\title{
The Effect of Varying Levels of Purified Condensed Tannins on Performance, Blood Profiles, Meat Quality and Methane Emission by Male Bapedi Sheep Fed a Grass Hay and Sheep Pellet-Based Diet
}

Jones $\mathrm{Ng}$ 'ambi

University of Limpopo Faculty of Science and Agriculture

Morongoa Selapa

University of Limpopo Faculty of Science and Agriculture

David Brown

University of Limpopo Faculty of Science and Agriculture

Tlou Grace Manyelo ( $\square$ manyelo.t.g@gmail.com )

University of Limpopo https://orcid.org/0000-0001-6394-8913

\section{Research Article}

Keywords: Bapedi sheep, Condensed tannins, Sheep performance, Meat quality, Methane emission

Posted Date: February 18th, 2022

DOI: https://doi.org/10.21203/rs.3.rs-1306426/v1

License: (c) (i) This work is licensed under a Creative Commons Attribution 4.0 International License.

Read Full License 


\section{Abstract}

This study determined the effect of purified condensed tannin inclusion levels in a diet on production, haematological indices, blood biochemical components, meat quality and methane emission by yearling indigenous male Bapedi sheep on a grass hay and sheep pellet-based diet in a 28-day trial. The diets contained similar $(P>0.05)$ nutrients but with different $(P<0.05)$ purified condensed tannin supplementation levels. A complete randomized design was used. Twenty-four yearling male Bapedi sheep were assigned to four dietary treatments having different purified condensed tannin levels of 0 $\left(\mathrm{GH}_{80} \mathrm{P}_{20} \mathrm{PCT}\right.$ ) $, 30\left(\mathrm{GH}_{80} \mathrm{P}_{20} \mathrm{PCT}_{30}\right), 40\left(\mathrm{GH}_{80} \mathrm{P}_{20} \mathrm{PCT}_{40}\right)$ and $50\left(\mathrm{GH}_{80} \mathrm{P}_{20} \mathrm{PCT}_{50}\right) \mathrm{g} / \mathrm{kg} \mathrm{DM}$. A quadratic type of equation was also used to determine condensed tannin supplementation levels for optimal performance and methane emission reduction by sheep. Supplementing diets with purified condensed tannins did not affect $(P>0.05)$ intake, digestibility and performance of male Bapedi sheep. Supplementing diets with purified condensed tannins had no effect $(P>0.05)$ on blood components of male Bapedi sheep. Bapedi sheep meat $\mathrm{pH}$ and sensory attributes were not influenced $(P>0.05)$ by purified condensed tannin level in the diet. However, supplementing diets with purified condensed tannins decreased $(P<0.05)$ methane emission by 51 to $60 \%$. A 49.08g supplementation level with purified condensed tannins per kg DM diet was calculated, with the use of quadratic equations, to result in the lowest methane emission by male Bapedi sheep. Meat of male Bapedi rams on diets containing 30,40 or $50 \mathrm{~g}$ of purified condensed tannins per $\mathrm{kg}$ DM contained higher $(\mathrm{P}<0.05)$ antioxidant activities than meat from rams fed a feed without purified condensed tannins. These results indicate that purified condensed tannin supplementation levels used did not have adverse effects on Bapedi sheep. However, supplementation levels of 30,40 or $50 \mathrm{~g}$ of purified condensed tannins per $\mathrm{kg}$ DM diet reduced methane emission by 51 to $60 \%$, and increased sheep meat antioxidant activity values. Supplementing diets with purified condensed tannins has potential to reduce methane production and emission by sheep. However, long-term studies are recommended to ascertain the present findings.

\section{Introduction}

Bapedi sheep, an indigenous South African sheep breed, are nutritionally, economically and culturally very important in South Africa (Matlebyane, 2005). It is important for milk, meat, hide, and skin (Snyman, 2014). However, methane $\left(\mathrm{CH}_{4}\right)$ produced by ruminants adversely contributes to climate change (Lassey, 2007; Kaufmann et al., 2006). About 80 million tons of methane is produced annually, where $47 \%$ of those emissions is contributed by agriculture and $39 \%$ of that is emitted by ruminants through the digestive processes (Eckard et al., 2010; Gerber et al., 2013). Eighty-five percent of methane produced by ruminants is from enteric fermentation, with cattle, buffalos and small ruminants contributing 77,13 and $10 \%$, respectively (Gerber et al., 2013). Methane emission results in a $12 \%$ loss of energy intake by ruminants (Johnson and Johnson, 1995). Tannins in a diet inhibit ruminal ammonia and methane production during microbial fermentation (Carulla et al., 2005). Low tannin intake levels reduce methane production and emission without any adverse effects (Makkar et al., 2007). Various studies indicate that dietary tannins improve meat quality (Ngambu et al., 2012, 2013; Bakare and Chimonyo, 2011). However, 
Mapiye et al. (2010) and Mapiye et al. (2009) observed no differences in meat quality of Nguni cattle fed diets differing in tannin inclusion levels. Tannins are anti-nutritional factors and, hence, when taken in large amounts they can be toxic to the animal (Olafadehan et al., 2014). Such effects are manifested in haematological and biochemical indices (Taiwo and Anosa, 1995). However, data on purified condensed tannin supplementation levels for optimal methane emission reduction, meat quality and blood profiles of Bapedi sheep is not extensive and conclusive. This study, therefore, focused on the effect of supplementation level of purified condensed tannins in a diet on production, haematological indices, blood biochemical components, meat quality and methane emission by yearling male Bapedi sheep fed a diet based on grass hay and sheep pellets. Such information is useful in formatting strategies for improving sheep production and reducing their methane emission.

\section{Methodology}

\section{Experimental design, treatments and data collection}

This experiment was done at the University Experimental Farm (latitude $27.55^{\circ} \mathrm{S}$ and longitude $24.77^{\circ} \mathrm{E}$ ). The area is semi-arid, and the vegetation is mainly of grass species with mixtures of shrubs and trees. The shrubs are mainly tanniniferous Acacia species (Shiringani, 2007). Twenty-four yearling male Bapedi sheep, weighing $25 \pm 1.6 \mathrm{~kg}$, were assigned to four dietary treatments with three replicates and each replicate having two sheep. A completely randomized design was used. The diets had different purified condensed tannin supplementation levels of $0\left(\mathrm{GH}_{80} \mathrm{P}_{20} \mathrm{PCT}_{0}\right), 30\left(\mathrm{GH}_{80} \mathrm{P}_{20} \mathrm{PCT}_{30}\right), 40\left(\mathrm{GH}_{80} \mathrm{P}_{20} \mathrm{PCT}_{40}\right)$ and $50\left(\mathrm{GH}_{80} \mathrm{P}_{20} \mathrm{PCT}_{50}\right) \mathrm{g} / \mathrm{kg} \mathrm{DM}$ (Table 1). The experimental diets were isocaloric and isonitrogenous. The experiment lasted for 28 days (21-day preliminary and 7-day collection periods). The sheep were accommodated in well-ventilated individual metabolic pens $(1.4 \mathrm{~m} \times 2.0 \mathrm{~m})$ with separate compartments for faecal and urine collections. Feed was offered ad libitum, allowing a 15\% refusal (Kaitho et al., 1996). The purified condensed tannins were purchased from Bondite Limited, South Africa. The animals were offered ad libitum water and a mineral lick (Table 2). An ethical clearance certificate (Certificate No: AREC/07/2019:PG) was obtained before the start of the experiment.

Daily diet intakes and weekly live weights was determined during the collection period (McDonald et al., 2011). All faeces were collected and apparent nutrient digestibility determined during the collection period (McDonald et al., 2011). Blood was collected from each Bapedi sheep jugular vein on the 21 and 28 days of the trial. A hand-held methane detector was used to measure methane emissions (Chagunda et al., 2009). Measurements were taken at $8.00 \mathrm{hrs}$ in the morning. Methane emitted was reported as parts per million-meter (ppm-m) (Chagunda et al., 2009).

All the sheep were slaughtered at the end of the experiment and their meat was subjected to $\mathrm{pH}$ and sensory attribute evaluations (Pavelková et al., 2013) to determine carcass characteristics. Sensory attributes of the meat were done according to the methods of Pavelková et al. (2013). 
Table 1

Dietary treatments for the study

\section{Experimental Treatment Description}

Code

$\mathrm{GH}_{80} \mathrm{P}_{20} \mathrm{PCT}_{0} \quad$ A mixture of $80 \%$ grass hay and $20 \%$ pellets without any purified condensed tannins.

$\mathrm{GH}_{80} \mathrm{P}_{20} \mathrm{PCT}_{30} \quad$ A mixture of $80 \%$ grass hay and $20 \%$ pellets plus $30 \mathrm{~g}$ of purified condensed tannins/kg DM feed.

$\mathrm{GH}_{80} \mathrm{P}_{20} \mathrm{PCT}_{40} \quad$ A mixture of $80 \%$ grass hay and $20 \%$ pellets plus $40 \mathrm{~g}$ of purified condensed tannins/kg DM feed.

$\mathrm{GH}_{80} \mathrm{P}_{20} \mathrm{PCT}_{50} \quad$ A mixture of $80 \%$ grass hay and $20 \%$ pellets plus $50 \mathrm{~g}$ of purified condensed tannins/kg DM feed. 
Table 2

Nutrient composition of the mineral block

\begin{tabular}{|ll|}
\hline Nutrients & Quantity \\
\hline Protein (g/kg DM) & 210 \\
\hline Urea (g/kg DM) & 20 \\
\hline Crude fibre (g/kg DM) & 99 \\
\hline Calcium (g/kg DM) & 20 \\
\hline Phosphorus (g/kg DM) & 5 \\
\hline Moisture (g/kg) & 130 \\
\hline Sulphur (g/kg DM) & 8 \\
\hline Potassium (g/kg DM) & 12 \\
\hline Copper (mg/kg DM) & 15 \\
\hline Manganese (mg/kg DM) & 100 \\
\hline Zinc (mg/kg DM) & 120 \\
\hline Cobalt (mg/kg DM) & 0.5 \\
\hline lodine (mg/kg DM) & 3 \\
\hline Iron (mg/kg DM) & 75 \\
\hline Selenium (mg/kg DM) & 0.5 \\
\hline Vitamin A (IU/kg) & 88. \\
\hline Energy (MJ/kg DM) & 8.8 \\
\hline Source: Voermol Maxi Block, South Africa \\
\hline
\end{tabular}

\section{Chemical Analysis}

AOAC (2012) procedures were used to determine nutrient composition of the diets. A bomb calorimeter was used to determine gross energy of diets and faeces (AOAC, 2012). Butanol-HCl method was used to determine condensed tannins (Makkar et al., 1995). The methods of Natt and Herrick (1952) and Mukkur and Bradley (1974) were used to determine total white blood cells and lymphocyte population, respectively. The procedures outlined by Dacie and Lewis (2001) were used to determine haematocrit and haemoglobin concentrations. Red blood cells and white blood cells were determined by Neubauer haemocytometer. The methods of Valley et al. (1980) were used to determine serum protein. Blood glucose was determined by enzymatic colorimetric test kit (Quimica Clinica Applicada, SA Kit). An atomic absorption spectrophotometer (Model 490, Gallenkamp and Co. Ltd., London) was used to estimate 
mineral contents in feeds and blood. The methods of Reitman and Frankel (1957) and Roy (1970) were used to measure the activities of the enzymes. The 2, 2-diphenyl-1-picrylhydrazyl (DPPH) method was used to estimate antioxidant activities in meat (Sgherri et al., 2012).

\section{Data analysis}

One-way analysis of variance procedures of SAS version 9.1.3 (SAS, 2008) were used. $Y_{i j}=\mu+T_{i}+e_{i j}$, where $Y_{i j}=$ overall observation, $\mu$ = overall means, $T_{i}=$ treatment effect and $e_{i j}=$ residual error. Differences between treatments were separated by LSD test $(P<0.05$; SAS 2008). The quadratic equation was used to determine purified condensed tannin level for optimal methane reduction by Bapedi sheep (SAS, 2008):

$Y=a+b_{1} x+b_{2} x^{2}+e$

Where $y=$ overall observation; $a=$ intercept; $b_{1}$ and $b_{2}=$ coefficients of the quadratic equation; $x=$ value of purified condensed tannin inclusion; $-b_{1} / 2 b_{2}=$ purified condensed tannin supplementation level for optimal response; and e = error.

\section{Results}

The nutrient contents of the diets were similar $(P>0.05)$ except for purified condensed tannins $(P<0.05)$ (Table 3). Supplementation with purified condensed tannins increased $(P<0.05)$ condensed tannin contents of the diets.

Supplementing diets with purified condensed tannins did not influence $(P>0.05)$ intake, digestibility and growth of yearling male Bapedi sheep (Table 4). However, supplementing diets with purified condensed tannins decreased $(P<0.05)$ methane emission by male Bapedi sheep. A 49.08g supplementation level with purified condensed tannins per $\mathrm{kg}$ DM diet was calculated, with the use of quadratic equations, to result in the lowest methane emission by male Bapedi sheep $\left(Y=23.882+-0.58883978 x+0.006066 x^{2} ; r^{2}\right.$ $=0.999$ ). The supplementation levels of $0,30,40$ or $50 \mathrm{~g}$ of purified condensed tannins per $\mathrm{kg} \mathrm{DM}$ diet used in this study did not affect $(P>0.05)$ haematological indices, blood metabolites and electrolytes of yearling male Bapedi sheep (Table 5). Supplementation with $0,30,40$ or $50 \mathrm{~g}$ of purified condensed tannins per kg DM did not affect $(\mathrm{P}>0.05)$ Bapedi sheep meat $\mathrm{pH}$ and sensory attributes (Table 6).

The effects of supplementing diets with purified condensed tannins on antioxidant activity in meat of male Bapedi ram are presented in Table 7. Meat from male Bapedi rams on diets containing 30, 40 or $50 \mathrm{~g}$ of purified condensed tannins per $\mathrm{kg}$ DM had better $(P<0.05)$ antioxidant activities than those from rams on a feed without purified condensed tannins. However, meat samples of male Bapedi rams fed diets having 30,40 or $50 \mathrm{~g}$ of purified condensed tannins per $\mathrm{kg}$ DM had the same $(P>0.05)$ antioxidant activities. 
Table 3

Nutrient composition of diets

\begin{tabular}{|c|c|c|c|c|}
\hline \multirow[t]{2}{*}{ Nutrient (\%) } & \multicolumn{4}{|l|}{ Diet $^{\# *}$} \\
\hline & $\mathrm{GH}_{80} \mathrm{P}_{20} \mathrm{PCT}_{0}$ & $\mathrm{GH}_{80} \mathrm{P}_{20} \mathrm{PCT}_{30}$ & $\mathrm{GH}_{80} \mathrm{P}_{20} \mathrm{PCT}_{40}$ & $\mathrm{GH}_{80} \mathrm{P}_{20} \mathrm{PCT}_{50}$ \\
\hline Dry matter (\%) & $95^{\mathrm{a}} \pm 0.53$ & $94^{\mathrm{a}} \pm 0.70$ & $95^{\mathrm{a}} \pm 0.55$ & $95^{\mathrm{a}} \pm 0.57$ \\
\hline Organic matter (\%) & $86^{a} \pm 0.52$ & $86^{a} \pm 0.07$ & $86^{a} \pm 0.44$ & $86^{a} \pm 0.45$ \\
\hline Crude protein (\%) & $12.5^{\mathrm{a}} \pm 0.65$ & $13^{\mathrm{a}} \pm 0.58$ & $13^{a} \pm 0.53$ & $13^{\mathrm{a}} \pm 0.51$ \\
\hline NDF (\%) & $45^{\mathrm{a}} \pm 0.48$ & $45^{\mathrm{a}} \pm 0.03$ & $45^{\mathrm{a}} \pm 0.25$ & $45^{\mathrm{a}} \pm 0.21$ \\
\hline ADF (\%) & $35^{\mathrm{a}} \pm 0.23$ & $35^{\mathrm{a}} \pm 0.44$ & $35^{\mathrm{a}} \pm 0.32$ & $35^{a} \pm 0.38$ \\
\hline Fat (\%) & $2.8^{a} \pm 0.31$ & $2.8^{a} \pm 0.25$ & $2.8^{\mathrm{a}} \pm 0.13$ & $2.8^{\mathrm{a}} \pm 0.26$ \\
\hline Energy (MJ/kg DM) & $18.3^{\mathrm{a}} \pm 0.26$ & $18.4^{\mathrm{a}} \pm 0.19$ & $18.2^{\mathrm{a}} \pm 0.21$ & $18.7^{\mathrm{a}} \pm 0.32$ \\
\hline Condensed tannins ${ }^{\neq}$ & $0^{d} \pm 0.00$ & $30^{c} \pm 0.01$ & $40^{\mathrm{b}} \pm 0.03$ & $50^{\mathrm{a}} \pm 0.02$ \\
\hline \multicolumn{5}{|c|}{${ }^{\star}$ : Values presented as mean \pm standard error (SE). } \\
\hline \multicolumn{5}{|c|}{$a, b, c, d$ : Means in the same row not sharing a common superscript are different $(P<0.05)$} \\
\hline \multicolumn{5}{|c|}{ \# : Treatments were supplementation with $0\left(\mathrm{GH}_{80} \mathrm{P}_{20} \mathrm{PCT}_{0}\right) 30\left(\mathrm{GH}_{80} \mathrm{P}_{20} \mathrm{PCT}_{30}\right), 40$} \\
\hline \multicolumn{5}{|c|}{$\left(\mathrm{GH}_{80} \mathrm{P}_{20} \mathrm{PCT}_{40}\right)$ or $50\left(\mathrm{GH}_{80} \mathrm{P}_{20} \mathrm{PCT}_{50}\right) \mathrm{g}$ of purified condensed tannins per kg DM } \\
\hline
\end{tabular}

diet.

$\neq: 0,30,40$ or $50 \mathrm{~g}$ of condensed tannins per $\mathrm{kg}$ DM diet. 
Table 4

Effect of supplementing diets with purified condensed tannins on intake, digestibility, methane emission and performance of male Bapedi rams

\begin{tabular}{|c|c|c|c|c|}
\hline & Diet $^{\# *}$ & & & \\
\hline Variable & $\mathrm{GH}_{80} \mathrm{P}_{20} \mathrm{PCT}_{0}$ & $\mathrm{GH}_{80} \mathrm{P}_{20} \mathrm{PCT}_{30}$ & $\mathrm{GH}_{80} \mathrm{P}_{20} \mathrm{PCT}_{40}$ & $\mathrm{GH}_{80} \mathrm{P}_{20} \mathrm{PCT}_{50}$ \\
\hline \multicolumn{5}{|l|}{ DM Intake (g/sheep/day) } \\
\hline Dry matter & $265 \pm 63.89$ & $338 \pm 40.30$ & $368 \pm 40.48$ & $287 \pm 40.95$ \\
\hline Organic matter & $230 \pm 52.77$ & $297 \pm 34.95$ & $320 \pm 41.70$ & $250 \pm 34.01$ \\
\hline Crude protein & $18.9 \pm 8.21$ & $25.5 \pm 3.30$ & $30.1 \pm 4.29$ & $25.0 \pm 3.60$ \\
\hline NDF & $161 \pm 29.15$ & $195 \pm 22.97$ & $214 \pm 26.51$ & $166 \pm 22.40$ \\
\hline ADF & $109 \pm 30.95$ & $128 \pm 19.96$ & $151 \pm 23.67$ & $110 \pm 25.85$ \\
\hline Fat & $5.2 \pm 0.90$ & $5.1 \pm 0.58$ & $5.1 \pm 0.77$ & $5.1 \pm 0.55$ \\
\hline Energy intake (MJ/day) & $5.6 \pm 0.71$ & $6.0 \pm 0.68$ & $6.5 \pm 0.72$ & $5.1 \pm 0.69$ \\
\hline \multicolumn{5}{|l|}{ Intake $\left(\mathrm{g} / \mathrm{kgW}^{0.75}\right)$} \\
\hline Dry matter & $35 \pm 17.52$ & $53 \pm 3.81$ & $55 \pm 8.67$ & $46 \pm 10.78$ \\
\hline Organic matter & $33 \pm 14.48$ & $49 \pm 7.53$ & $50 \pm 8.47$ & $43 \pm 10.04$ \\
\hline Crude protein & $4.7 \pm 2.07$ & $7 \pm 0.24$ & $7 \pm 1.36$ & $6 \pm 1.47$ \\
\hline NDF & $16 \pm 7.24$ & $26 \pm 2.89$ & $26 \pm 3.29$ & $21 \pm 5.01$ \\
\hline ADF & $11 \pm 4.17$ & $17 \pm 2.58$ & $17 \pm 2.83$ & $14 \pm 3.36$ \\
\hline Fat & $1.1 \pm 0.811$ & $1.1 \pm 0.741$ & $2.2 \pm 0.571$ & $1.2 \pm 0.712$ \\
\hline Energy intake $\left(\mathrm{MJ} / \mathrm{kg} \mathrm{W}^{0.75}\right)$ & $0.91 \pm 0.091$ & $1.1 \pm 0.064$ & $1.1 \pm 0.085$ & $1.1 \pm 0.174$ \\
\hline \multicolumn{5}{|l|}{ Digestibility (decimal) } \\
\hline Dry matter & $0.6 \pm 0.15$ & $0.6 \pm 0.07$ & $0.6 \pm 0.08$ & $0.4 \pm 0.28$ \\
\hline Organic matter & $0.6 \pm 0.16$ & $0.6 \pm 0.07$ & $0.6 \pm 0.09$ & $0.4 \pm 0.27$ \\
\hline Crude protein & $0.4 \pm 0.35$ & $0.4 \pm 0.12$ & $0.4 \pm 0.13$ & $0.2 \pm 0.48$ \\
\hline NDF & $0.5 \pm 0.20$ & $0.4 \pm 0.09$ & $0.6 \pm 0.11$ & $0.2 \pm 0.38$ \\
\hline ADF & $0.3 \pm 0.29$ & $0.3 \pm 0.12$ & $0.4 \pm 0.15$ & $0.1 \pm 0.53$ \\
\hline${ }^{*}:$ Mean \pm standard error $(\mathrm{SE})$ & & & & \\
\hline
\end{tabular}




\begin{tabular}{|lllll|}
\hline \multicolumn{5}{|c|}{ Diet $^{\# *}$} \\
\hline Fat & $0.7 \pm 0.52$ & $0.4 \pm 0.20$ & $0.4 \pm 0.29$ & $0.4 \pm 0.92$ \\
\hline Energy & $0.5 \pm 0.18$ & $0.5 \pm 0.08$ & $0.6 \pm 0.09$ & $0.4 \pm 0.34$ \\
\hline Methane emission (ppm-m) & $23.9^{\mathrm{a}} \pm 2.80$ & $11.5^{\mathrm{b}} \pm 1.02$ & $10.3^{\mathrm{b}} \pm 1.04$ & $9.5^{\mathrm{b}} \pm 1.02$ \\
\hline Live weight (g/sheep/day) & & & \\
\hline Initial & $25.0 \pm 0.67$ & $25.1 \pm 0.58$ & $24.9 \pm 2.03$ & $24.8 \pm 2.67$ \\
\hline Final & $25.7 \pm 2.31$ & $25.8 \pm 0.33$ & $25.6 \pm 4.36$ & $25.5 \pm 6.00$ \\
\hline Wt. gain(g/sheep/day) & $25 \pm 0.33$ & $25 \pm 0.88$ & $25 \pm 0.33$ & $25 \pm 0.60$ \\
\hline Feed conversion ratio & $11 \pm 2.33$ & $13 \pm 1.8$ & $14 \pm 2.8$ & $12 \pm 1.33$ \\
\hline * : Mean \pm standard error (SE) & & & & \\
\hline a, b $:$ Means in the same row not sharing a common superscript are significantly different \\
\hline
\end{tabular}

$(P<0.05)$.

\# : Treatments were supplementation with $0\left(\mathrm{GH}_{80} \mathrm{P}_{20} \mathrm{PCT}_{0}\right) 30\left(\mathrm{GH}_{80} \mathrm{P}_{20} \mathrm{PCT}_{30}\right), 40$

$\left(\mathrm{GH}_{80} \mathrm{P}_{20} \mathrm{PCT}_{40}\right)$ or $50\left(\mathrm{GH}_{80} \mathrm{P}_{20} \mathrm{PCT}_{50}\right) \mathrm{g}$ of purified condensed tannins per kg DM

diet 
Table 5

Effects of supplementing diets with purified condensed tannins on blood components of Bapedi rams

\begin{tabular}{|c|c|c|c|c|}
\hline \multirow[t]{2}{*}{ Variable } & \multicolumn{4}{|l|}{$\operatorname{Diet}^{\# *}$} \\
\hline & $\mathrm{GH}_{80} \mathrm{P}_{20} \mathrm{PCT}_{0}$ & $\mathrm{GH}_{80} \mathrm{P}_{20} \mathrm{PCT}_{30}$ & $\mathrm{GH}_{80} \mathrm{P}_{20} \mathrm{PCT}_{40}$ & $\mathrm{GH}_{80} \mathrm{P}_{20} \mathrm{PCT}_{50}$ \\
\hline Red blood cell $\left(\times 10^{12} / \mathrm{L}\right)$ & $1.7 \pm 4.91$ & $2.4 \pm 0.86$ & $2.0 \pm 0.78$ & $2.0 \pm 0.33$ \\
\hline Haemoglobin (g/dL) & $9.4 \pm 0.92$ & $9.9 \pm 0.93$ & $9.7 \pm 0.95$ & $8.4 \pm 0.92$ \\
\hline Haematocrit (L/L) & $0.1 \pm 0.03$ & $0.1 \pm 0.03$ & $0.1 \pm 0.03$ & $0.1 \pm 0.03$ \\
\hline White blood cell $\left(\times 10^{9} / \mathrm{L}\right)$ & $18.5 \pm 10.6$ & $19.8 \pm 6.54$ & $29.6 \pm 7.72$ & $17.5 \pm 6.89$ \\
\hline \multicolumn{5}{|l|}{ Metabolites (mmol/L) } \\
\hline Urea & $3.3 \pm 0.62$ & $3.4 \pm 0.58$ & $4.03 \pm 0.69$ & $2.5 \pm 0.92$ \\
\hline Glucose & $1.7 \pm 1.02$ & $2.8 \pm 0.30$ & $2.89 \pm 0.21$ & $3.0 \pm 0.19$ \\
\hline Cholesterol & $3.3 \pm 0.84$ & $2.8 \pm 0.18$ & $2.42 \pm 0.13$ & $2.5 \pm 0.12$ \\
\hline \multicolumn{5}{|l|}{ Proteins (g/L) } \\
\hline Total protein & $75.0 \pm 2.82$ & $71.3 \pm 2.54$ & $68.7 \pm 2.50$ & $70.0 \pm 2.50$ \\
\hline Albumin & $24.5 \pm 1.97$ & $26.0 \pm 1.97$ & $25.0 \pm 2.01$ & $28.0 \pm 2.00$ \\
\hline \multicolumn{5}{|l|}{ Enzymes (IU/L) } \\
\hline Alkaline phosphate & $63.0 \pm 30.98$ & $85.0 \pm 31.20$ & $108 \pm 29.52$ & $84.0 \pm 30.45$ \\
\hline Alanine transaminase & $16.5 \pm 2.39$ & $18.7 \pm 1.83$ & $17.0 \pm 1.75$ & $19.7 \pm 1.94$ \\
\hline Aspartate transaminase & $65.0 \pm 7.46$ & $69.0 \pm 5.26$ & $69.3 \pm 6.30$ & $73.3 \pm 5.83$ \\
\hline \multicolumn{5}{|l|}{ Electrolytes (mmol/L) } \\
\hline Sodium & $143 \pm 1.32$ & $145 \pm 1.42$ & $143 \pm 1.56$ & $146 \pm 1.33$ \\
\hline Potassium & $7.6 \pm 0.61$ & $6.7 \pm 1.61$ & $7.6 \pm 0.49$ & $7.1 \pm 0.49$ \\
\hline Chloride & $110 \pm 1.72$ & $111 \pm 1.52$ & $114 \pm 2.41$ & $112 \pm 1.32$ \\
\hline Total calcium & $2.2 \pm 0.06$ & $2.2 \pm 0.06$ & $2.2 \pm 0.06$ & $2.2 \pm 0.06$ \\
\hline Inorganic phosphate & $1.8 \pm 0.15$ & $1.8 \pm 0.14$ & $2.0 \pm 0.15$ & $1.9 \pm 0.16$ \\
\hline \multicolumn{5}{|c|}{${ }^{*}:$ Mean \pm standard error $(\mathrm{SE})$} \\
\hline \multicolumn{5}{|c|}{ \# : Treatments were supplementation with $0\left(\mathrm{GH}_{80} \mathrm{P}_{20} \mathrm{PCT}_{0}\right) 30\left(\mathrm{GH}_{80} \mathrm{P}_{20} \mathrm{PCT}_{30}\right), 40$} \\
\hline \multicolumn{5}{|c|}{$\left(\mathrm{GH}_{80} \mathrm{P}_{20} \mathrm{PCT}_{40}\right)$ or $50\left(\mathrm{GH}_{80} \mathrm{P}_{20} \mathrm{PCT}_{50}\right) \mathrm{g}$ of purified condensed tannins per $\mathrm{kg} \mathrm{DM}$} \\
\hline
\end{tabular}




\begin{tabular}{|c|c|c|c|c|}
\hline \multirow[t]{2}{*}{ Variable } & \multicolumn{4}{|l|}{ Diet $^{\# *}$} \\
\hline & $\mathrm{GH}_{80} \mathrm{P}_{20} \mathrm{PCT}_{0}$ & $\mathrm{GH}_{80} \mathrm{P}_{20} \mathrm{PCT}_{30}$ & $\mathrm{GH}_{80} \mathrm{P}_{20} \mathrm{PCT}_{40}$ & $\mathrm{GH}_{80} \mathrm{P}_{20} \mathrm{PCT}_{50}$ \\
\hline Magnesium & $1.0 \pm 0.06$ & $1.0 \pm 0.06$ & $1.0 \pm 0.06$ & $1.1 \pm 0.07$ \\
\hline \multicolumn{5}{|c|}{${ }^{*}:$ Mean \pm standard error (SE). } \\
\hline \multicolumn{5}{|c|}{ \# : Treatments were supplementation with $0\left(\mathrm{GH}_{80} \mathrm{P}_{20} \mathrm{PCT}_{0}\right) 30\left(\mathrm{GH}_{80} \mathrm{P}_{20} \mathrm{PCT}_{30}\right), 40$} \\
\hline \multicolumn{5}{|c|}{$\left(\mathrm{GH}_{80} \mathrm{P}_{20} \mathrm{PCT}_{40}\right)$ or $50\left(\mathrm{GH}_{80} \mathrm{P}_{20} \mathrm{PCT}_{50}\right) \mathrm{g}$ of purified condensed tannins per kg DM } \\
\hline
\end{tabular}

diet. 
Table 6

Effect of supplementing diets with purified condensed tannins on Bapedi ram meat attributes

\begin{tabular}{|c|c|c|c|c|}
\hline \multirow[t]{2}{*}{ Variable } & \multicolumn{4}{|l|}{ Diet $^{\neq \#}$} \\
\hline & $\mathrm{GH}_{80} \mathrm{P}_{20} \mathrm{PCT}_{0}$ & $\mathrm{GH}_{80} \mathrm{P}_{20} \mathrm{PCT}_{30}$ & $\mathrm{GH}_{80} \mathrm{P}_{20} \mathrm{PCT}_{40}$ & $\mathrm{GH}_{80} \mathrm{P}_{20} \mathrm{PCT}_{50}$ \\
\hline \multicolumn{5}{|l|}{$\mathrm{pH}$} \\
\hline Breast & $5.9 \pm 0.11$ & $6.0 \pm 0.01$ & $6.0 \pm 0.01$ & $5.9 \pm 0.21$ \\
\hline Rump & $5.7 \pm 0.02$ & $5.6 \pm 0.11$ & $5.7 \pm 0.01$ & $5.7 \pm 0.01$ \\
\hline Shank & $5.4 \pm 0.02$ & $5.4 \pm 0.01$ & $5.4 \pm 0.01$ & $5.3 \pm 0.11$ \\
\hline Shoulder & $5.6 \pm 0.11$ & $5.6 \pm 0.10$ & $5.8 \pm 0.11$ & $5.7 \pm 0.09$ \\
\hline \multicolumn{5}{|l|}{ Meat colour (rump) } \\
\hline Lightness & $49.3 \pm 0.96$ & $47.7 \pm 0.85$ & $48.0 \pm 0.77$ & $49.0 \pm 0.82$ \\
\hline Redness & $10.3 \pm 0.10$ & $8.7 \pm 0.10$ & $15.3 \pm 0.10$ & $14.3 \pm 0.10$ \\
\hline Yellowness & $5.7 \pm 0.95$ & $5.8 \pm 0.81$ & $5.7 \pm 0.71$ & $6.3 \pm 0.90$ \\
\hline \multicolumn{5}{|l|}{ Meat Attributes } \\
\hline Tenderness & $3.7 \pm 1.53$ & $4.3 \pm 1.58$ & $4.0 \pm 1.30$ & $3.3 \pm 1.15$ \\
\hline Juiciness & $3.3 \pm 0.58$ & $3.0 \pm 1.00$ & $3.3 \pm 0.58$ & $3.3 \pm 0.58$ \\
\hline Flavour & $3.6 \pm 0.33$ & $3.7 \pm 0.33$ & $3.7 \pm 0.33$ & $3.7 \pm 0.33$ \\
\hline Overall acceptability & $4.0 \pm 0.02$ & $4.0 \pm 0.08$ & $4.0 \pm 0.03$ & $4.0 \pm 0.03$ \\
\hline Shear force & $29.7 \pm 4.04$ & $32.5 \pm 2.30$ & $31.8 \pm 1.42$ & $29.9 \pm 2.06$ \\
\hline \multicolumn{5}{|c|}{$\neq:$ Mean \pm standard error $(\mathrm{SE})$} \\
\hline \multicolumn{5}{|c|}{ \# : Treatments were supplementation with $0\left(\mathrm{GH}_{80} \mathrm{P}_{20} \mathrm{PCT}_{0}\right) 30\left(\mathrm{GH}_{80} \mathrm{P}_{20} \mathrm{PCT}_{30}\right), 40$} \\
\hline
\end{tabular}

diet. 
Table 7

Antioxidant activities in meat extracts of male Bapedi rams using DPPH assay

\begin{tabular}{|ll|}
\hline Treatment* & Antioxidant activity \\
\hline $\mathrm{GH}_{80} \mathrm{P}_{20} \mathrm{PCT}_{0}$ & $20.6^{\mathrm{b}} \pm 1.89$ \\
\hline $\mathrm{GH}_{80} \mathrm{P}_{20} \mathrm{PCT}_{30}$ & $31.1^{\mathrm{a}} \pm 2.99$ \\
\hline $\mathrm{GH}_{80} \mathrm{P}_{20} \mathrm{PCT}_{40}$ & $36.1^{\mathrm{a}} \pm 2.96$ \\
\hline $\mathrm{GH}_{80} \mathrm{P}_{20} \mathrm{PCT}_{50}$ & $31.2^{\mathrm{a}} \pm 2.98$ \\
\hline $\mathrm{a}, \mathrm{b},:$ Means in the same column not sharing a common superscript are significantly \\
\hline
\end{tabular}

different $(P<0.05)$.

* : Treatments were supplementation with $0\left(\mathrm{GH}_{80} \mathrm{P}_{20} \mathrm{PCT}\right) 30\left(\mathrm{GH}_{80} \mathrm{P}_{20} \mathrm{PCT}_{30}\right), 40$

$\left(\mathrm{GH}_{80} \mathrm{P}_{20} \mathrm{PCT}_{40}\right)$ or $50\left(\mathrm{GH}_{80} \mathrm{P}_{20} \mathrm{PCT}_{50}\right) \mathrm{g}$ of purified condensed tannins per $\mathrm{kg} \mathrm{DM}$

diet.

\# : Mean \pm standard error (SE).

\section{Discussion}

Diets contained similar protein and energy levels of $13 \%$ and $18 \mathrm{MJ} / \mathrm{kg} \mathrm{DM}$, respectively. The diets met recommended nutrient requirements for slow-growing sheep (NRC, 2007). Diets had similar nutrient contents except purified condensed tannins ranging from 0 to $50 \mathrm{~g} / \mathrm{kg} \mathrm{DM}$. These diets were, therefore, appropriate for the determination of condensed tannin levels for optimal performance and reduced methane emission in yearling male Bapedi sheep. It was expected that the diets would have similar intake and digestibility values (McDonald et al., 2011) provided that condensed tannin supplementation did not have any adverse effects. Indeed, supplementing diets with purified condensed tannins did not affect intake, digestibility and weight gain of male Bapedi sheep. It is possible that the purified condensed tannin amounts were not too high to adversely affect intake, digestibility and weight gain of male Bapedi sheep. Al-Dobaib (2009) also reported that lambs fed diets having different levels of quebracho tannins had similar intakes and weight gains. However, other studies showed a decrease in intake and digestibility of nutrients when condensed tannins were incorporated into the ruminant diets (Makkar, 2007). The binding ability of tannins to microorganism cell walls adversely affects enzyme secretion and nutrient transportation within the organisms (McSweeney et al., 2001); thus, nutrient digestibility decreases because rumen microorganisms are not able to optimally ferment the diets. However, other studies found that condensed tannin inclusions in the diets had beneficial effects on intake and weight gain of ruminant animals (Min et al., 2003; Soltan et al., 2012). 
Diet supplementation with purified condensed tannins reduced methane emission by Bapedi rams, by 51 to $60 \%$. A $49.08 \mathrm{~g}$ supplementation of purified condensed tannins per $\mathrm{kg}$ DM diet was calculated, with the use of quadratic equations, to optimize methane emission reduction by male Bapedi sheep. This indicates that condensed tannin levels used in the study were enough to inhibit methanogenesis (Makkar et al., 2007). Carulla et al. (2005) observed a $12 \%$ reduction in methane emission when sheep were fed diets having different levels of condensed tannins. The authors indicated that tannins reduced methane production by decreasing synthesis of acetate during digestion. Woodward et al. (2001) reported a 20 to $24 \%$ decrease in methane emission when sheep were fed diets containing condensed tannins. The present study indicates that purified condensed tannin supplementation levels of 30,40 and $50 \mathrm{~g}$ per $\mathrm{kg}$ DM diet resulted in similar methane emission levels by sheep; possibly meaning that the levels used were within the required effective amounts for inhibition of methanogens in sheep. Mbanzamihigo et al. (2002) observed similar methane emission amounts by sheep when fed diets with tanniniferous legume supplementation levels of $0.16,0.35,0.41$ or $0.51 \mathrm{~g} / \mathrm{kg}$ DM.

Nutritional problems can be detected in the blood of animals (Ajao, 2013). In the present study, purified condensed tannin supplementation levels of $0,30,40$ or $50 \mathrm{~g}$ per $\mathrm{kg}$ DM did not affect blood parameters of male Bapedi sheep. Amounts of purified condensed tannins used induced no adverse effects in the animals. Haemoglobin values observed are normal for a health sheep (Daramola et al., 2005; Akinyemi et al., 2010; Akinrinmade and Akinrinde, 2012). The present results findings are like those of Dey et al. (2008) and Pathak et al. (2013). Purified condensed tannin supplementations did not affect haematocrit values of male Bapedi sheep, indicating that the animals were not anaemic (Purves et al., 2003; Ndlovu et al., 2007). Solaiman et al. (2010) and Brown et al. (2016) observed similar results in goats on diets differing in tannin amounts.

There were no significant variations in white blood cell (WBC) counts of Bapedi sheep on diets differing in purified condensed tannin supplementation levels, indicating that the animals were in good health (Ahamefule et al., 2008; Solaiman et al., 2010). Brown et al. (2016) observed similar results in Bapedi goats on diets differing in tannins.

Purified condensed tannin supplementation did not affect blood urea values of male Bapedi sheep. The blood urea values are within the range for a health sheep (Daramola et al., 2005; Akinyemi et al., 2010). Serum glucose values of male Bapedi sheep were not affected by dietary supplementation with purified condensed tannins, and they are within the normal range for a health sheep (Naumann et al., 2017; Pathak et al., 2013; Midaoui and de Champlain, 2005).

Blood cholesterol values were similar among Bapedi sheep fed diets with different levels of purified condensed tannins. These cholesterol levels, ranging from 2.42 to $3.3 \mathrm{mmol} / \mathrm{L}$, were within the normal values for a healthy sheep (Naumann et al., 2017; Siri-Tarino et al., 2010). Olafadehan et al. (2014) observed similar cholesterol values when goats were on diets high in condensed tannins. Supplementation with purified condensed tannins did not affect blood protein indices of male Bapedi sheep. The blood protein indices were normal for a healthy sheep (Naumann et al., 2017; Jain, 1986). 
Purified condensed tannin inclusion in the diet had no effect on serum enzyme levels of Bapedi rams, suggesting that the animals were in good health (Thapa and Walia, 2007). Dey et al. (2008) observed no effects of tanniniferous Ficus infectoria leaf meal supplementation on aspartate transaminase and alanine transaminase levels in lambs. Purified condensed tannin supplementation levels did not affect blood electrolytes and mineral values of Bapedi rams. The electrolytes and mineral values obtained were normal for healthy sheep (Naumann et al. (2017), possibly meaning that the amounts used did not affect mineral absorption in the gut of the animals (Naumann et al. (2017; Sowande et al., 2008).

Condensed tannin supplementation did not adversely affect male Bapedi meat $\mathrm{pH}$, sensory attributes and shear force values. The amounts $0,30,40$ or $50 \mathrm{~g}$ of purified condensed tannins per $\mathrm{kg}$ DM diet did not affect the end products of digestion and hence no differences in meat attributes of the sheep were expected. Dentinho et al. (2020) observed similar results when lambs were fed diets having different tannin levels. Similarly, Brown and Ngámbi (2018) observed no differences in goat meat attributes when diets differing in Vachelia karroo leaf meals were fed to goats. However, Luzardo et al. (2019) and Luciano et al. (2009) observed that tanniniferous feeds improved meat colour of sheep. Gesteira et al. (2018) concluded that lamb meat tenderness decreased and shear force values increased as dietary tannin levels increased. Kobue-Lekalake et al. (2009) and Priolo et al. (2000) observed that meat of lambs on diets with lower tannin levels had better taste. The present study indicates that purified condensed tannin supplementation levels did not affect Bapedi sheep meat $\mathrm{pH}$ values which were within the range for a healthy sheep (Mostert, 2007). Priolo et al. (2002), also, observed that tannin supplementation had no effect on sheep meat $\mathrm{pH}$ values. However, other authors reported increased meat $\mathrm{pH}$ values when sheep were supplemented with purified condensed tannins (Ngambu et al., 2013).

Purified condensed tannin supplementation levels of 30,40 or $50 \mathrm{~g} / \mathrm{kg}$ DM diet increased antioxidant activity values in ram meat. Sufficient uptake of dietary antioxidants has been observed as an effective method to prevent lipid oxidation and avoid oxidation of cells and tissues caused by excess free radicals and oxidative stress (Negukhula, 2010). High antioxidant activity values in meat increase the quality and shelf-life of meat by reducing lipid oxidation (Fernandez-Gines et al., 2005; Karami et al., 2011; Juarez et al., 2012; Kasapidou et al., 2012; Karre et al., 2013). Marume et al. (2012) found that Diet supplementation with tanniniferous Acacia karroo leaf meal improved goat meat sensory attributes (Marume et al., 2012). Velasco and Williams (2011) also reported that chicken meat sensory attributes can be improved by inclusion of antioxidants in the diets.

\section{Conclusion}

Supplementing diets with $0,30,40$ or $50 \mathrm{~g}$ of purified condensed tannins per $\mathrm{kg}$ DM did not affect intake, digestibility and growth of yearling Bapedi rams, possibly indicating that the amounts of tannins used had no adverse effects on the animals. This can be attributed to the fact that the diets were iso-energetic and iso-nitrogenous. The supplementation levels of $0,30,40$ or $50 \mathrm{~g}$ of purified condensed tannins per $\mathrm{kg}$ DM diet did not affect haematological parameters, blood metabolites and electrolytes, possibly indicating 
that the levels used were not toxic to the rams. However, long term experiments are suggested to ascertain the present findings.

Supplementing diets with 30,40 or $50 \mathrm{~g}$ of purified condensed tannins per $\mathrm{kg}$ DM reduced methane emission by Bapedi rams by 51 to $60 \%$. A $49.08 \mathrm{~g}$ supplementation level with purified condensed tannins per kg DM diet was calculated to optimize methane emission reduction by Bapedi rams. These results indicate that condensed tannin levels used in the study were enough to inhibit methanogenesis and hence reduce methane production and emission. However, further long-term studies are suggested to ascertain the ruminal pathways. Purified condensed tannin supplementation levels of $0,30,40 \mathrm{or} 50 \mathrm{~g}$ of purified condensed tannins per $\mathrm{kg}$ DM did not adversely affect sheep meat $\mathrm{pH}$ and sensory attributes. However, dietary supplementation levels of 30,40 or $50 \mathrm{~g}$ of purified condensed tannins per $\mathrm{kg}$ DM increased antioxidant activity values in ram meat, possibly indicating improved meat shelf-life (Karre et al., 2013).

It is concluded that condensed tannin supplementation levels of $0,30,40$ or $50 \mathrm{~g} / \mathrm{kg}$ DM diet did not affect diet digestibility, growth and Bapedi ram meat sensory attributes. However, condensed tannin supplementation levels used in this study increased antioxidant activity values in male Bapedi ram meat. Long term studies are suggested to ascertain the present results.

\section{Declarations}

Funding: Thanks to NRF and VLIR-UOS (grant number ZIUS2016AP21) for funding this study.

Conflicts of Interest: No conflict of interest.

Ethics approval: The study was ethically cleared (Certificate No: AREC/07/2019:PG).

Consent to participate: Not applicable.

Availability of data and materials: Available.

Code of availability: N/A

Acknowledgements: Authors would like to thank University of Limpopo Experimental Farm workers for their help and cooperation during the trial.

Authors' contribution: JWN: conceptualization, visualization, funding acquisition, manuscript writing and editing; MJS: data collection and writing-original draft; DB: data analysis and manuscript editing; TGM: data analysis, manuscript review and manuscript editing.

\section{References}


1. Ahamefule, F.O., Obua, B.E., Ukweni, M.A., Oguike, M.A., Amaka, R.A., 2008. Haematological and biochemical profile of weaned rabbits fed raw or processed pigeon seed meal-based diets. African Journal of Agricultural Research, 3, 315-319.

2. Ajao, B.H., Ola, S.I., Adameji, O.V., Kolawole, R.F., 2013. The relationship of ambient temperature and relative humidity to the thermo respiratory function of greater grass cutter. Proceedings of the $18^{\text {th }}$ Annual Conference of Animal Science Association of Nigeria. pp. 92.

3. Akinrinmade, J.F., Akinrinde A.S., 2012. Haematological and serum biochemical indices of West African dwarf goats with foreign body rumen impaction. Nigerian Journal of Physiological Sciences, 27, 83-87.

4. Akinyemi, A.F., Alokan, J.A., Fajemisin, A.N., 2010. Digestibility, nitrogen balance and haematological profile of West African Dwarf sheep fed dietary levels of Moringa oleifera as supplement to Panicum maximum. The Journal of American Science, 6, 634-643.

5. Al-Dobaib, S.N., 2009. Effect of different levels of quebracho tannin on nitrogen utilization and growth performance of Najdi sheep fed alfalfa (Medicago sativa) hay as a sole diet. Journal of Animal Science. 80, 532-541.

6. AOAC, 2012. Official methods of analysis. 18th edition. Association of Official Analytical Chemists, Arlington, Virginia, USA.

7. Bakare, A.G., Chimonyo, M., 2011. Seasonal variation in time spent foraging by indigenous goat genotypes in a semi-arid rangeland in South Africa. Livestock Science, 135, 251-256.

8. Bray, A.R., Graafbuis, A.E., Chrystall, B.B., 2001. The cumulative effect of nutritional, shearing and pre slaughter washing stresses on the quality of lamb meat. Meat Science, 25, 59-67.

9. Brown, D., Ng'ambi, J.W., Norris, D., Mbajiorgu, F.E., 2016. Blood profiles of indigenous Pedi goats fed varying levels of Vachellia karroo leaf meal in Setaria verticillata hay-based diet. South African Journal of Animal Science, 46 (4), 432-440.

10. Carulla, J.E., Kreuzer, M., Machmüller, A., Hess, H.D., 2005. Inclusion of Acacia mearnsii tannins decreases methanogenesis and urinary nitrogen in forage-fed sheep. Australian Journal of Agricultural Research, 56, 961-970.

11. Chagunda, M.G.G., Römer, D.A.M., Roberts, D.J., 2009. Effect of genotype and feeding regime on enteric methane, non-milk nitrogen and performance of dairy cows during the winter feeding period. Livestock Science, 122, 323-332.

12. Dacie, J.V., Lewis, S.M., 2001. Practical Haematology $9^{\text {th }}$ ed. Churchill Livingstone, London, ed. Churchill Livingstone, London, pp. 633.

13. Daramola, J.O., Adeloye, A.A., Fatoba, T., Soladoye, A.O., 2005. Haematological and biochemical parameters of West African Dwarf goats. Livestock Research and Rural Development, 17, 8-11

14. Dentinho, T.P., Paulos, K., Francisco, A., Belo, A.T., Jerónimo, E., Almeida, J., Bessa, R.J.B., SantosSilva, J., 2020. Effect of soybean meal treatment with Cistus ladanifer condensed tannins in growth performance, carcass and meat quality of lambs. Livestock Science, 236, 104021-104032. 
15. Dey, A., Dutta, N., Sharma, K., Pattanaik, A.K., 2008. Effect of dietary inclusion of Ficus infectoria leaves as a protectant of proteins on the performance of lambs. Small Ruminant and Research. 75, 105-114.

16. Dubey, M., Dutta, N., Banerjee, P.S., Pattanaik, A.K., Sharma, K., Singh, M., 2012. Effect of condensed tannins supplementation through a tree leaves mixture on erythrocytic antioxidant status and gastrointestinal nematodes in kids. Animal Nutrition and Feed Technology, 12, 91-102.

17. Eckard, R.J., Grainger, C., Klein, C.A.M., 2010. Options for the abatement of methane and nitrous oxide from ruminant production. A review. Livestock Science, 130, 47-56.

18. Fernández-Ginés, J. M., Fernández-López, J., Sayas-Barberá, E. and Pérez-Alvarez, J. (2005). Meat products as functional foods: Review. Journal of Food Science, 70(2), 37-43.

19. Gerber, P.J., Steinfeld, H., Henderson, B., Motet, A., Opie, C., Felucca, A., Tempie, G., Kyriakakis, I., 2013. Tackling climate change through livestock: a global assessment of emissions and mitigation opportunities. Food and Agriculture Organization of the United Nations (FAO), Rome, Italy.

20. Gesteira, S.M., Oliveira, R.L., Silva, T.M., Ribeiro, R.D.X., Cláudio V.D.M Ribeiro, C.V.D.M., Pereira, E.S., Lanna, D.P.D., Pinto, L.F.B., Rocha, T.C., Vieira, J.F., Leilson, R.B., Bezerra, L.R., 2018. Physicochemical quality, fatty acid composition, and sensory analysis of Nellore steers meat fed with inclusion of condensed tannins in the diet. Food Science, 83(5), 1366-1372.

21. Jain, N.C. 1986. Schalm Veterinary Hematology, $4^{\text {th }}$ ed. Lea and Febiger, Philadelphia, USA

22. Johnson, K.A., Johnson, D.E., 1995. Methane emissions from cattle. Journal of Animal Science, 73, 2483-2492.

23. Juárez, M., Dugan, M. E., Aldai, N., Basarab, J. A., Baron, V. S., McAllister, T. A. and Aalhus, J. L. 2012. Beef quality attributes as affected by increasing the intramuscular levels of vitamin $\mathrm{E}$ and omega-3 fatty acids. Meat Science 90(3): 764-769.

24. Karami, M., Alimon, A.R. and Goh, Y.M. 2011. Effect of vitamin E, Andrographis paniculata and turmeric as dietary antioxidant supplementation on lipid and color stability of goat meat. Small Ruminant Research, 97, 67-71.

25. Karre, L.; Lopez, K.; Getty, J.K. 2013. Natural antioxidants in meat and poultry products. Meat Science, $94,220-227$.

26. Kasapidou, E., Wood, J.D., Richardson, R.I., Sinclair, L.A., Wilkinson, R.G. and Enser, M. 2012. Effect of vitamin E supplementation and diet on fatty acid composition and on meat colour and lipid oxidation of lamb leg steaks displayed in modified atmosphere packs. Meat Science, 90(4), 908916.

27. Kaufmann, R.K., Kauppi, H., Stock, J.H., 2006. Emissions, concentrations, and temperature: A time series analysis. Nature Climate Change, 77, 249-278.

28. Kobue-Lekalake, R., Taylor, J.R.N., de Kock, H.L., 2009. Influence of PROP taster status on the consumer acceptability of food made from tannin sorghums. Journal of the Science of Food and Agriculture, 89, 11. 
29. Lassey, K.R., 2008. Livestock methane emission and its perspective in the global methane cycle. Australian Journal of Experimental Agriculture, 48, 114-118.

30. Luciano, G., Monahan, F.J., Vasta, V., Biondi, L., Lanza, M., Priolo, A., 2009. Dietary tannins improve lamb meat colour stability. Meat Science, 81, 120-12.

31. Luzardo, S., Mederos, A., Brito G., 2019. Effect of condensed tannins in Lotus uliginosus cv. E-Tannin and/or extracts of quebracho and chestnut in carcass and lamb meat. Meat Science, 23(1), 1-8.

32. Makkar, H.P.S. 2003. Effects and fate of tannins in ruminant animals, adaptation to tannins, and strategies to overcome detrimental effects of feeding tannin-rich feeds. Small Ruminant Research, $49,241-56$.

33. Makkar, H.P.S., Blümmel, M., Becker, K., 1995. In vitro effects and interactions of tannins and saponins and fate of tannins in rumen. Journal of the Science of Food and Agriculture, 69, 481-493.

34. Makkar, H.P.S., Franscis, G., Becker, K., 2007. Bioactivity of phytochemicals in some lesser-known plants and their effects and potential applications in livestock and aquaculture production systems. Animals, 1, 1371-1391.

35. Mapiye, C., Chimonyo, M., Dzama, K., Strydom, P. E., Marufu, M. C., Muchenje, V., 2009. Nutritional status, growth performance and carcass characteristics of Nguni steers supplemented with Acacia karroo leaf-meal. Livestock Science, 126, 206-214.

36. Mapiye, C., Chimonyo, M., Dzama, K., Strydom, P. E., Muchenje, V., 2010. Meat quality attributes of Nguni steers supplemented with Acacia karroo leaf-meal. Meat Science, 8, 621-627.

37. Marume, U., Chimonyo, M. and Dzama, K. 2012. Influence of dietary supplementation with Acacia karroo on experimental haemonchosis in indigenous Xhosa lop-eared goats of South Africa. Livestock Science, 144,132-139.

38. Matlebyane, M.M., 2005. Relationship between chemical composition, in vitro digestibility and locally based feeding value rankings and medicinal use of some common forages for ruminant livestock in three area of the Capricorn of Limpopo Province, South Africa. M.Sc. Dissertation, School of Agricultural and Environmental Sciences, Faculty of Sciences, Health and Agriculture. University of Limpopo, South Africa.108p.

39. Mbanzamihigo, L., Fievez, V., da Costa Gomez, C., Piattoni, F., Carlier, L., Demeyer, D., 2002. Methane emission from the rumen of sheep fed a mixed grass-clover pasture at two fertilisation rates in early and late season. Canadian Journal of Animal Science, 82, 69-77.

40. McDonald, P., Edwards, R.A., Greenhalgh, J.F.D., Morgan, C.A., Sinclair, L.A., Wilkinson, R.G., 2011. Nutrient digestion and the environment. In Animal Nutrition, 7th ed., Blackwell, London, UK. pp. 188189.

41. McSweeney, C.S., Palmer, B., McNeill, D.M., Krause, D.O., 2001. Microbial interactions with tannins: nutritional consequences for ruminants. Animal Feed Science and Technology, 9, 83-93.

42. Midaoui, A.E.I., de Champlain, J., 2005. Effects of glucose and insulin on the development of oxidative stress and hypertension in animal models of type 1 and type 2 diabetes. Journal of Hypertension, 23 (3), 581-588. 
43. Min, B.R., Barry, T.N., Attwood, G.T., McNabb, W.C., 2003. The effect of condensed tannins on the nutrition and health of ruminants fed fresh temperate forages: a review. Animal Feed Science and Technology, 106, 3-19.

44. Mostert, A.C., 2007. Meat quality of kudu (Tragelaphus strepsiceros) and impala (Aepyceros melampus). MSc Thesis, University of Stellenbosch, South Africa.

45. Mukkur, T.K.S. and Bradley, R.E., 1974. Determination of the absolute numbers of leukocyte cell types in chicken. Poultry science, 53, 221-223.

46. Natt, M.P. and Herrick, C.A., 1952. A new diluent for counting the erythrocytes and leukocytes of the chicken. Poultry Science, 31, 735-737.

47. Naumann, H.D., Tedeschi, L.O., Zeller, W.E., Huntley, N.F., 2017. The role of condensed tannins in ruminant animal production: advances, limitations and future directions. Zootechnology. 46(12), 3036.

48. Ndlovu, T., Chimonyo, M., Okoh, A.I., Muchenje, V., Dzama, K., Raats, J.G., 2007. Assessing the nutritional status of beef cattle: current practices and future prospects. African Journal of Biotechnology, 6, 2727-2734.

49. Negukhula, S. 2010. Effects of brewing temperature and duration on quality of black Tea (Camellia sinensis) and equal (50:50) combination of bush tea (Athrixia phylicoides dc.) And black tea. Mini dissertation for Master's Degree in Horticulture. School of Agricultural and Environmental Sciences. University of Limpopo, South Africa: 4-12.

50. Ngambu, S., Muchenje, V., Marume, U., 2012. The effect of Acacia karroo supplementation and thermal preparation on meat sensory characteristics of the indigenous Xhosa lop-eared goat genotype. African Journal of Biotechnology 11, 12878-12884.

51. Ngambu, S., Muchenje, V., Marume, U., 2013. Effect of Acacia karroo supplementation on growth, ultimate $\mathrm{pH}$, colour and cooking losses of meat from indigenous Xhosa Lop-eared goats. Asianaustralas Journal of Animal Science, 26, 128-133.

52. NRC, 2007. Nutrient Requirements of Small Ruminants: Sheep, Goats, Cervids, and New World amelids. Washington, DC: The National Academies Press, USA.

53. Nunez-Hernandez, G., Holecheck, J.L., Wallace, J.D., Galyean, M.L., Tembo, A., Valdez, R., Cardenas, M., 1989. Influence of native shrubs diets on nutritional status of goats: Nitrogen retention Journal of Range Management, 42, 228-232.

54. Obeidat, B.S., Alrababah, M.A., Abdullah, A.Y., Alhamad, M.N., Gharaibeh, M.A., Rababah, T.M., Ishmais, M.A.A., 2011. Growth performance and carcass characteristics of Awassi lambs fed diets containing carob pods (Ceratonia siliqua L.). Small Ruminant Research, 96(2-3), 149-154.

55. Olafadehan, O.A., Adewumi, M.K., Okunade, S.A., 2014. Effects of feeding tannin-containing forage in varying proportion with concentrate on the voluntary intake, haematological and biochemical indices of goats. Trakia Journal of Science, 1, 73-81.

56. Pathak, A.K., Dutta, N., Banerjee, P.S., Pattanaik, A.K., Sharma, K., 2013. Influence of dietary supplementation of condensed tannins through leaf meal mixture on intake, nutrient utilization and 
performance of Haemonchus contortus infected sheep. Asian-Australasia Journal of Animal Science, 26(10), 1446-1458.

57. Pavelková, A., Kačániová, M., Hleba, L., Petrová, J., Pochop, J., Čuboň, J., 2013. Sensory evaluation of chicken breast treated with Oregano essential oil. Journal of Animal Science and Biotechnology, 46(2), 379-383.

58. Priolo, A., Waghorn, G.C., Lanza, M., Biondi, L., Pennisi, P., 2000. Polyethylene glycol as a means for reducing the impact of condensed tannins in carob pulp: effects on lamb growth performance and meat quality. Journal of Animal Science, 78, 810-816.

59. Priolo, A., Ben Salem, H., Atti, N., Nefzaoui, A., 2002. Polyethylene glycol in concentrate or feedblocks to deactivate condensed tannins in Acacia cyanophylla Lindl. Foliage: effects on meat quality of Barbarine lambs. Animal Science, 75, 137-140.

60. Purves, W.K., Sadava, D., Orians, G.H., Heller, H.C., 2003. Life: The Science of Biology (7 $7^{\text {th }}$ ed.). Sinauer Associates and W.H. Freeman. pp. 954.

61. Reitman, S., Frankel, S., 1957. A colorimetric method for determination of serum glutamic oxaloacetic and transaminase serum glutamic pyruvic transaminase. American Journal of Clinical Pathology, 28, 56-63.

62. Roy, A.V., 1970. Rapid method for determining alkaline phosphatase activity in serum with thymolphthalein monophophatase. Clinical Chemistry. 16, 431-436.

63. SAS. 2008. Statistical Analysis System User's Guide: Statistics, 9th ed. SAS Institute, Raleigh, North Caroline, USA.

64. Sgherri, C., Pinzino, C. and Quartacci, M.F. 2012. Antioxidant potential in lipophilic and hydrophilic extracts from medicinal herbs (Salvia officinalis and Echinacea angustifolia). A comparison between assays based on electron paramagnetic resonance and spectrophotometry. American Journal of Agricultural and Biological Sciences, 7, 417-424.

65. Shiringani, R.P., 2007. Effect of planting date and location on phenology, yield and yield components among selected cowpea varieties. Master's dissertation. University of Limpopo, South Africa. pp. 20-73.

66. Siri-Tarino, P.W., Sun, Q., Hu, F.B. and Krauss, R.M., 2010. Meta-analysis of prospective cohort studies evaluating the association of saturated fat with cardiovascular disease. American Journal of Clinical Nutrition, 91 (3), 535-546.

67. Solaiman, S., Thomas, J., Dupre, Y., Min, B.R., Gurung, N., Terrill, T.H., Haenlein, G.F.W., 2010. Effect of feeding sericea lespedeza (Lespedeza cuneata) on growth performance, blood metabolites, and carcass characteristics of Kiko crossbred male kids. Small Ruminant Research, 93, 149-156.

68. Soltan, M.A., Mujalli, A.M., Mandour, M., Abeer, M., 2012. Effect of Dietary Rumen Protected Methionine and/or Choline Supplementation on Rumen Fermentation Characteristics and Productive Performance of Early Lactating Cows. Pakistan Journal of Nutrition, 11, 221-230. 10.3923/pjn.2012.221.230. 
69. Sowande, O.S., Odufowora, E.B., Adelakun, A.O., Egbeyale, L.T., 2008. Blood minerals in WAD sheep and goats grazing natural pastures during wet and dry seasons. Animal Archives, 57 (218), 275-278.

70. Snyman, M.A., 2014. South African Sheep Breeds: Pedi Info-pack ref. 2014/025. Grootfontein Agricultural Development Institute, South Africa.

71. Taiwo, V.O., Anosa, V.O., 1995. Fibrinogen, leucocyte and haematocrit values of cattle with various disease conditions. Tropical Veterinarian, 13, 51-57.

72. Thapa, B.R., Walia, A., 2007. Liver function tests and their interpretation. Indian Journal of Paediatrics, 74 (7), 663-671.

73. Velasco, V. and Williams, P. 2011. Improving meat quality through natural antioxidants. Chilean Journal of Agriculture Research, 71, 313-322.

74. Valley, H., Gowelock, A.H., Bell, M., 1980. Determination of serum urea using diacetyl monoxime method. In: Practical Clinical Biochemistry, 5 onoxime method. In: Practical Clinical Biochemistry, 5thed. William Heinemann Medical ed. William Heinemann Medical Books Ltd., London.

75. Van Soest, P.J., 1994. Nutritional Ecology of the Ruminants. 2nd Edn., Cornell University Press, New York, ISBN: 9780801427725, pp 476.

76. Woodward, S.L., Waghorn, G.C., Ulyatt, M.J., Lassey, K.R., 2001. Early indication that feeding lotus will reduce methane emission from ruminants. Proceedings of the New Zealand Society of Animal Production, 61: 23-26. 